\title{
2213
}

\section{The origins of modularity in complex machines}

\author{
Victor Parque ${ }^{* 1}$, Masakazu Kobayashi ${ }^{* 1}$, Masatake Higashi ${ }^{* 1}$ \\ ${ }^{* 1}$ Toyota Technological Institute \\ 2-12-1 Hisakata, Tempaku-ku, Nagoya 468-8511, Japan
}

\begin{abstract}
Modularity is vital to engineer complex products and machines. We assert that modularity can emerge from searching desirable machine structures constrained to life cycle costs in manufacturing, maintenance, and disassemblability. Compared to the conventional methods, computational experiments that maximize machine performance and minimize life cycle costs yield machine structures that are modular and tractable.
\end{abstract}

Key Words : Modularity, optimization, clustering

\section{Introduction}

We address the problem on how to find modules in a mechanical system given information of components in mesh structures(which represents the spatial structure of any mechanical system). Different from a mere connectivity-based community-finding problem, we formulate our approach with the goal of searching for a relevant organization (modular structures) that maximise pre-stated life cycle goals related to manufacturing, maintenance, and disassemblability.

Previous work in the arena has exploited the concept of clustering through diverse forms of modularity metrics(1)(2). The work in (1) showed that shape of the search space for the conventional clustering problem and asserted that there are more than one single solution that satisfies a maximal modularity index. The recent work in (2) offers insights on how to use concepts of clustering and fuzzy set for product and machine design.

From a practical point of view, the conventional clustering approach to modularity-search does not answer any question concerning life cycle performance, neither stakeholder satisfaction. For example, having more modules is important for costumers to enhance personalization, but having too many modules is detriment for manufacturers due to complexity(and lack of flexibility) of assembly procedures, thus eliminating economies of scale. In fact, given a modularity index, there is more than one single solution to the clustering problem(1).

For practical purposes, we propose a different approach for searching modules in complex mechanical systems, for which modules are also allowed to emerge. Inspired from (3) and (4), we model a system to search for a system structure that not only maximises performance, but also minimises costs in a given environment. The unique point of our approach is that wide applicability for practical mechanical systems, and the departure from the conventional view on community finding problem that implies searching for modules toward the structure-finding problem for mechanical systems.

The basic idea of our approach is to search over the space of organizational modules to optimise a metric of life cycle performance. Thus, modules emerge from searching such search space, thus targeting stakeholder satisfaction in the life cycle of the machine. Other practical life cycle metrics can be easily incorporated.

This paper is organized as follows: Section 2 details our approach, and Section 3 offers results on relevant study cases

\footnotetext{
"E-mail: vparque@toyota-ti.ac.jp
} 


\section{The origins of modularity in complex machines}

In order to search for modules, the input of our algorithm is a set $P=\left\{P_{l}, P_{2}, \ldots, P_{i}, \ldots, P_{n}\right\}$, where:

- $P$ represents the mechanical system.

- $P_{i}$ is the $i$-th component of $P$, represented by a mesh in $\mathbf{R}^{3}: P_{i}=\left(V_{i}, E_{i}\right)$ with node positions at $V_{i}$ and edges at $E_{i}$.

We aim at finding $P^{\prime}$, such that:

$$
\begin{aligned}
& f\left(P^{\prime}\right)=\sum_{s \in S} \lambda_{s}\left[\text { Performance }\left(s, P^{\prime}\right)-\operatorname{Cost}\left(s, P^{\prime}\right)\right] \\
& P^{\prime}=\bigcup_{k \in K} C\left(x_{k}\right)
\end{aligned}
$$

- $\quad P$ ' represents the modular organization of $P$.

- $S$ represents the set of stakeholders in the product life cycle of $P$. Basically, we assume the following $S=\{$ Consumer, Producer, Environment $\}$.

- $\quad \lambda_{s}$ represents the constant for the degree of importance of stakeholder $s$.

- $K$ represents the number of maximum allowed modules in $P(K<<n)$.

- $C\left(x_{k}\right)$ represents the set of components of $P$ belonging to module $k$.

- $x$ represents the solution vector to the modularity problem $\left(x \in[1, K]^{n}\right)$.

Basically, the above is an optimization problem, where we aim at finding $x$ such that it represents the modular organization of a mechanical structure $P$. Note that $P$ and $P^{\prime}$ are structurally equivalent. The role of $x$ is thus to allow organization and division of the components of $P$ into building blocks that maximize the utility for stakeholders in the life cycle of $P$. Next, we explicit how to assess the performance and the costs for the stakeholders assumed in this paper.

\section{$2 \cdot 1$ Costumers}

The performance and costs for consumers are assumed to be a function of personalization and repairing costs. Personalization is dependant of the number of modules and the number of options available per module. We use only the first factor as follows:

Performance (Consumer, $\left.P^{\prime}\right)=K$

On the other hand, repairing costs depend mainly on the number of parts per module. More parts imply higher repairing costs, and fewer parts imply the opposite. Unitary costs can be easily incorporated. The goal is to have a stable(low variability) and low number of parts per every module in $P^{\prime}$.

$$
\operatorname{Cost}\left(\text { Consumer }, P^{\prime}\right)=\bar{C} \cdot \sigma_{C}
$$

, where:

- $\bar{C}$ represents the average number of parts per every module in $P^{\prime}$.

- $\sigma_{C}$ represents the standard deviation of the number of parts per every module in $P^{\prime}$. 


\section{$2 \cdot 1$ Producers}

The advantage of having modular structures for manufacturers mainly lies on the compactness of the product and the economic performance for the assembly process. Conceptually, modules that are more compact have lower distance among its parts. Thus we aim at finding machine structures with modules that have a small and stable distance among its modules:

$$
\begin{aligned}
& \text { Performance }\left(\text { Producer, } P^{\prime}\right)=-\bar{T} . \sigma_{T} \\
& \bar{T}=\frac{1}{K} \sum_{k \in K} T\left(x_{k}\right) \\
& \bar{T}=\sqrt{\frac{1}{K} \sum_{k \in K}\left[T\left(x_{k}\right)-\bar{T}\right]^{2}} \\
& T\left(x_{k}\right)=D\left(x_{k}\right) \cdot \sigma\left(x_{k}\right) \\
& D\left(x_{k}\right)=\frac{1}{N\left(x_{k}\right)} \sum_{\forall P_{i}, P_{j} \in C\left(x_{k}\right), P_{i} \neq P_{j}} d\left(P_{i}, P_{j}\right)
\end{aligned}
$$

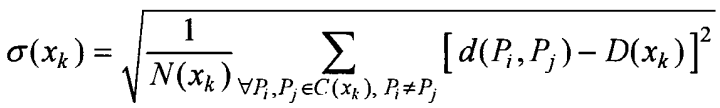

$$
\begin{aligned}
& N\left(x_{k}\right)=\left(\begin{array}{c}
\left|C\left(x_{k}\right)\right| \\
2
\end{array}\right)
\end{aligned}
$$

The above mainly computes the distance and variability of distance among parts inside every module for the machine. The distance $d\left(P_{i}, P_{j}\right)$ between the components $P_{i}$ and $P_{j}$ is the smallest distance between the vertexes $v_{i}$ and $v_{j}\left(v_{i} \in V_{i}, v_{j} \in V_{j}\right)$. Distance is pre-procesed by (5). In case of complex polyedra, we pre-process by brute search over the vertex sets in $V_{i}$ and $V_{j}$.

Similarly, the assembly costs is a function of the distance among parts in the modules. Close parts imply shorter wire lengths and less communication efforts among component parts. Furthermore, compact modules have lower coordination and assembly costs. Thus the assembly cost can be approximated from the total wire length in the product:

$$
\text { Cost (Producer, } \left.P^{\prime}\right)=\sum_{k \in K} \sum_{P_{i}, P_{j} \in C\left(x_{k}\right), P_{i} \neq P_{j}} d\left(P_{i}, P_{j}\right)
$$

\section{1 Environment}

Environmental factors such as reusability and dissasemblability are important in the life cycle of the machine $P$. Reusability depends on the average life expectancy and the complexity of a module. Highly durable materials and low complexity of module composition are highly reusable. Leaving the life expectancy factor for future work, we estimate the reusability of a module using the number of parts: fewer parts per module imply lower complexity, and more reusability of individual components. Thus, we aim at an averaged little number of parts per module in the machine $P$ '.

Performance (Environment, $\left.P^{\prime}\right)=-\bar{C} \cdot \sigma_{C}$

Similarly, dissasemblability implies efforts to break the connections among modules and parts inside every module. This effort depends on the number of modules and total number of parts in all the modules of $P$ ':

$$
\text { Cost (Environment, } \left.P^{\prime}\right)=K \sum_{k \in K}\left|C\left(x_{k}\right)\right|
$$




\section{Study Cases}

In order to evaluate the performance and scalability of our proposed approach, we use publicly available models(6) considering the different the number of $\operatorname{components}(n)$ and mesh compositions. The search algorithm to optimize Eq. (1) is performed by (7), using the parameters stated in the reference. The maximum number of objective evaluations is set to $1 \mathrm{e}^{+}+5$. The constant $\lambda_{s}$ representing the degree of importance of stakeholders is set at 1 for all cases. Adaptive parameter tuning is left for future work.

Fig. 1 to Fig. 5 show (a) the used models, (b) the connectivity graph resulting from the search for close distances and (c) the obtained modular structures. Table 1 summarizes the main results in terms of the objective $f$ in Eq. (1), the higher the better, together with the connectivity and module structure.
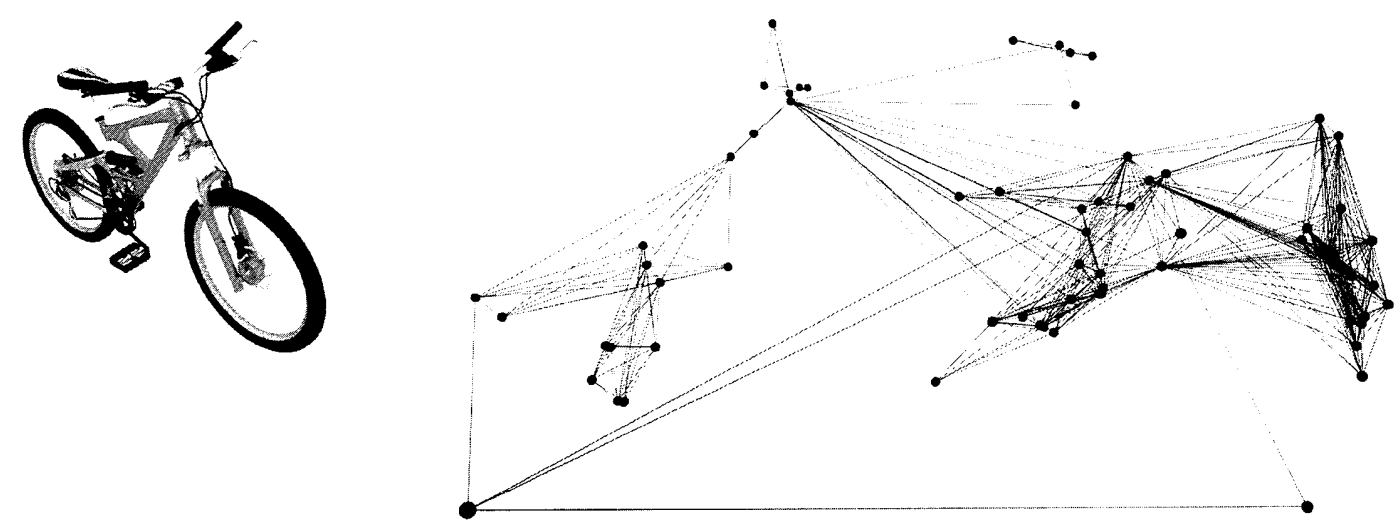

(a) Original Model

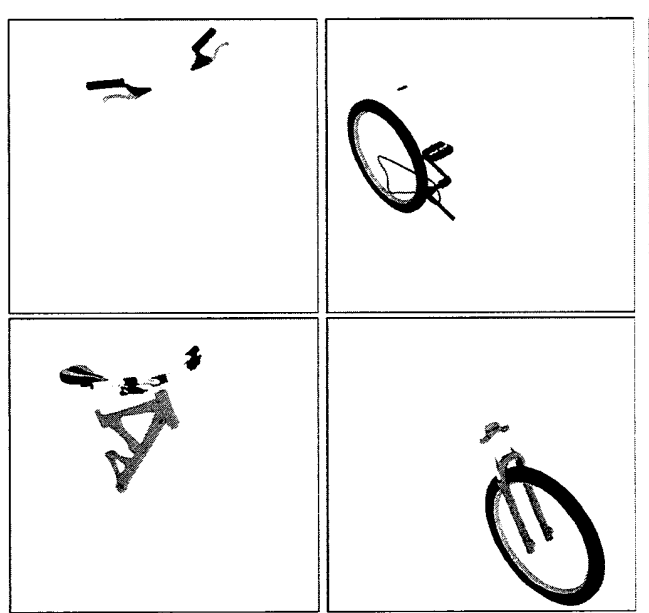

(b) Connectivity Graph

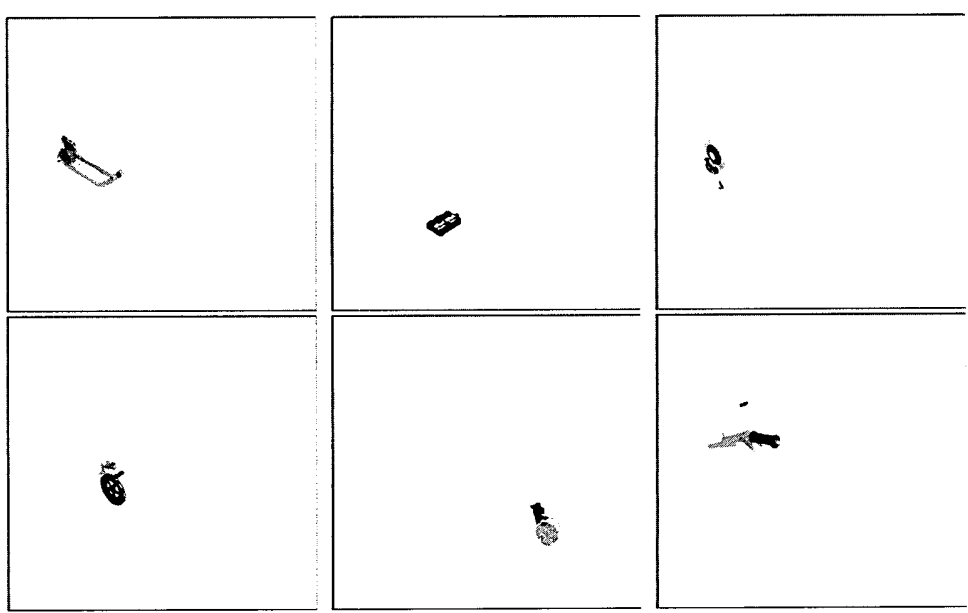

(c) Modular Structures

Fig. 1 Bicycle Model 


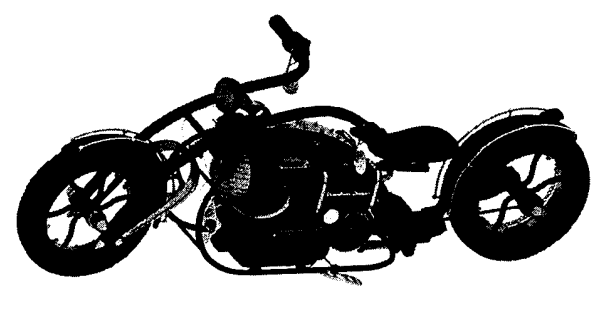

(a) Original Model
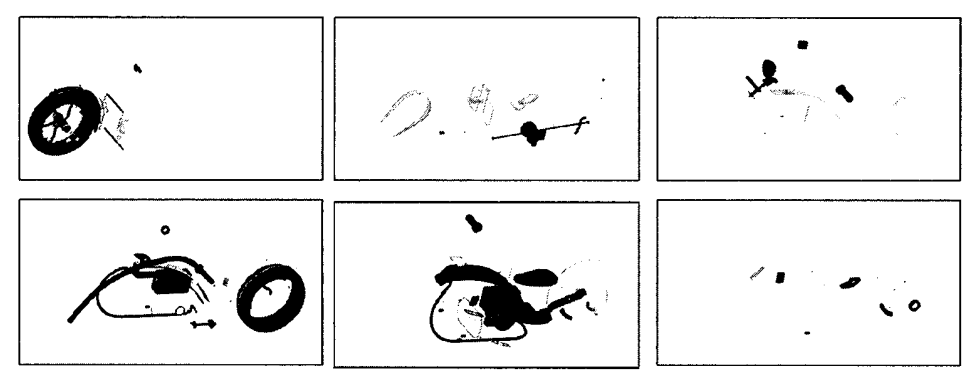

(c) Modular Structures

Fig. 2 Motorcycle Model

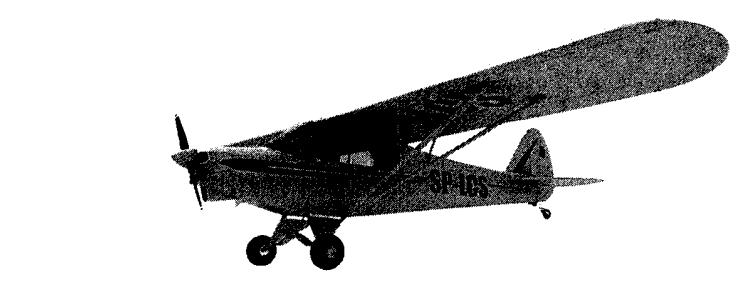

(a) Original Model

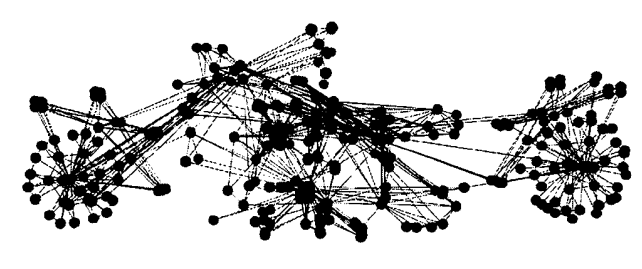

(b) Connectivity Graph

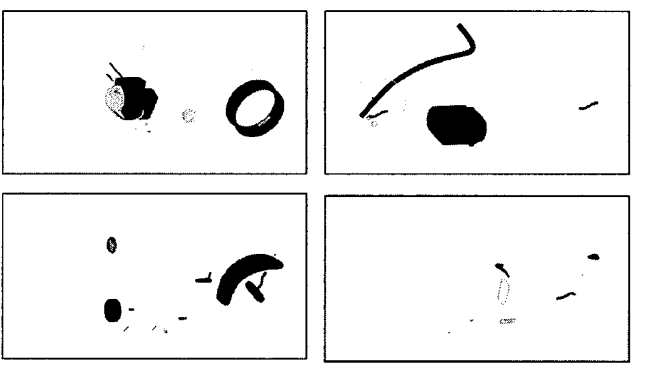

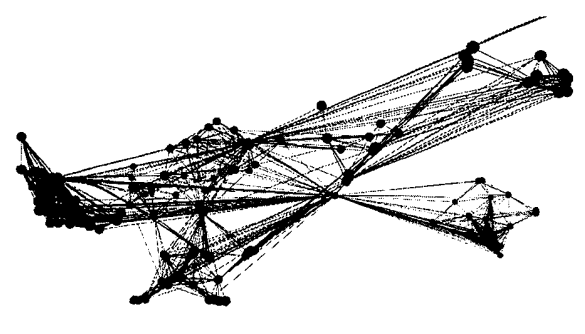

(b) Connectivity Graph

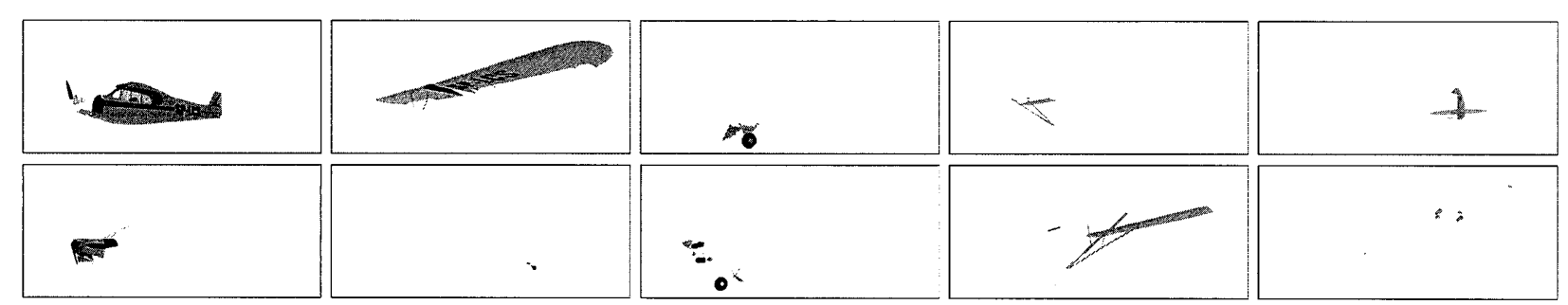

(c) Modular Structures

Fig. 3 Airplane Model 


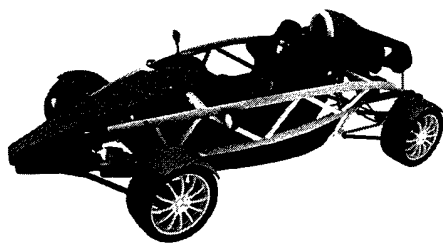

(a) Original Model

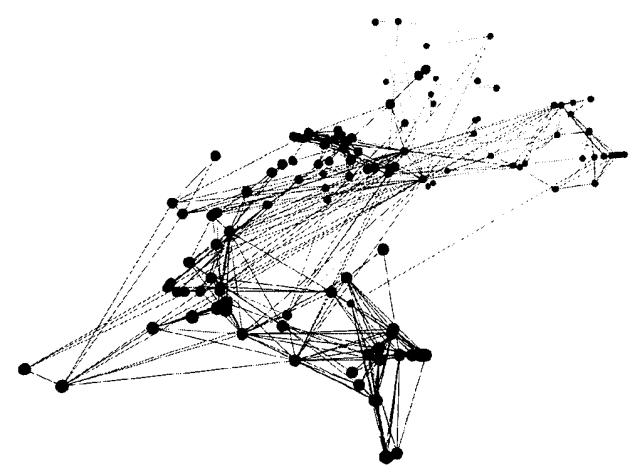

(b) Connectivity Graph
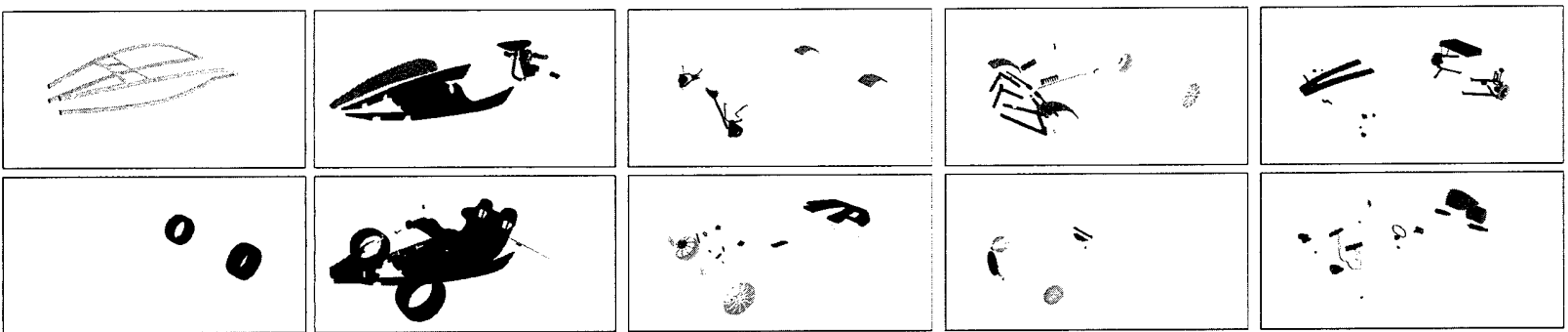

(c) Modular Structures

Fig. 4 Car Model

Table 1 Main study cases and results

\begin{tabular}{||c|c|c|c||}
\hline Model Name & $n$ & $f$ & Connectivity and Module Structure \\
\hline BICYCLE & 77 & -923.09 & Fig. 1 \\
\hline MOTORCYCLE & 304 & -9134.77 & Fig. 2 \\
\hline AIRPLANE & 192 & -3075.54 & Fig. 3 \\
\hline CAR & 287 & -5330.53 & Fig. 4 \\
\hline ROBOT & 469 & -22628.78 & Fig. 5 \\
\hline
\end{tabular}

The figure results represent the best cases of five independent runs. We can observe from Fig. 1- Fig. 5 that the proposed approach generates modules that are generally well divided and spatially compact. However, we can also observe that the models with higher number of components(MOTORCYCLE and ROBOT) have still very low objective values, thus requiring more fitness evaluations. Future work aims at studying the behavior with parameters and other search algorithms.

\section{Conclusion}

This paper has proposed a method to search for modular structures in complex machines. Different from a mere clustering problem, the proposed method can find modular structures by maximising machine life cycle costs. The applicability to practical design problems is straightforward and the future work aims at building modular machines from scratch. 


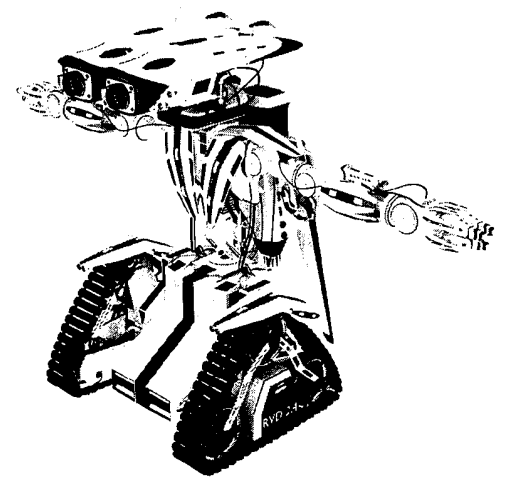

(a) Original Model

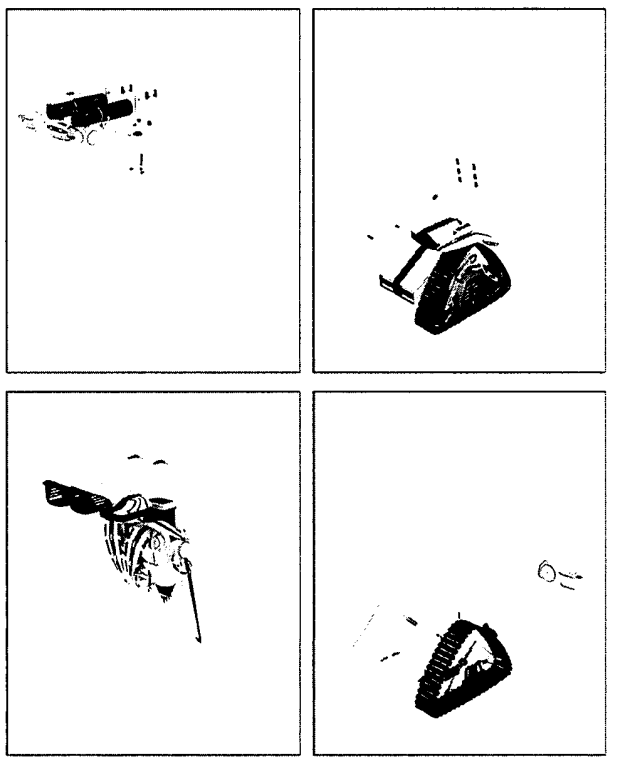

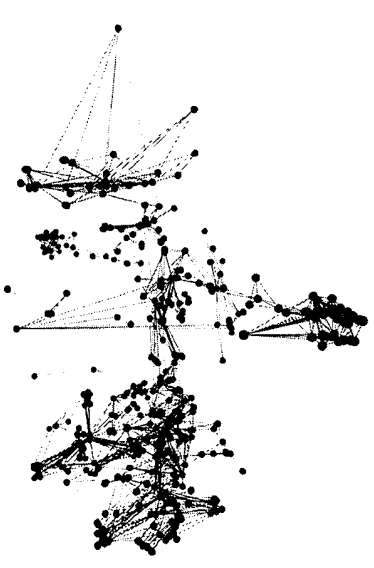

(b) Connectivity Graph

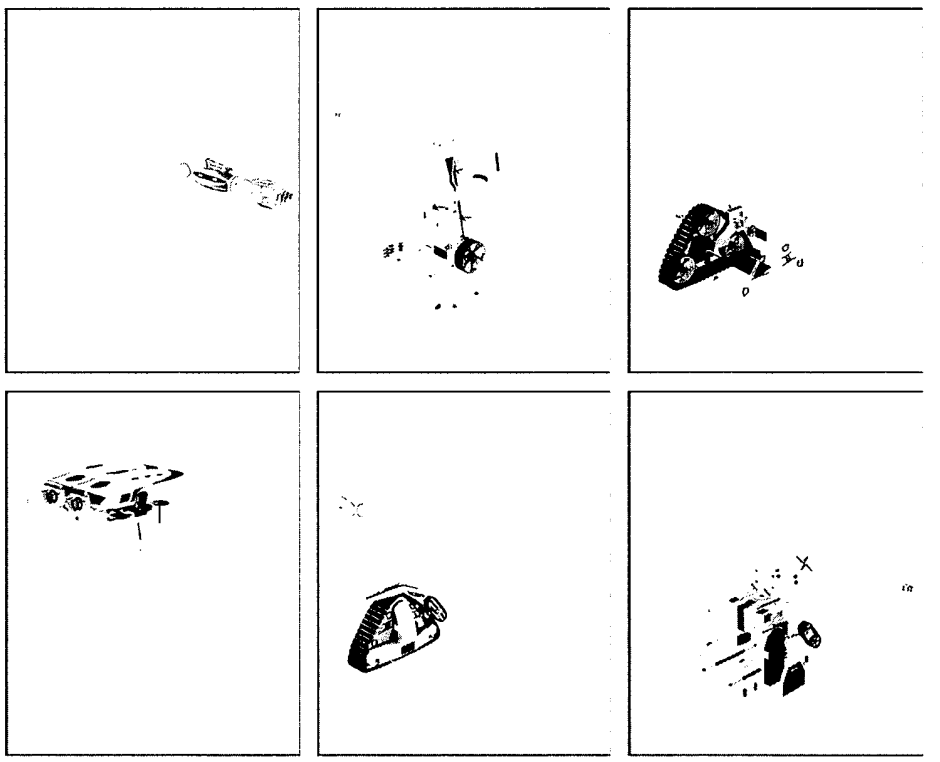

(c) Modular Structures

Fig. 5 Robot Model

\section{Bibliography}

(1) B. H. Good, Y. A. de Montjoye and A. Clauset., "The performance of modularity maximization in practical contexts", Physical Review, E 81, 046106 (2010).

(2) Z. Li, Z. Cheng, Y. Feng and J. Yang, "An integrated method for flexible platform modular architecture design", Journal of Engineering Design, Vol. 24, Issue 1, pp. 25-44, 2013.

(3) R. Jacobs and M. Jordan, "Computational consequences of a bias toward short connections", Journal of Cognitive Neuroscience, Vol. 4 (1992), pp. 323-336.

(4) J. Clune, J-B Mouret and H. Lipson, "The evolutionary origins of modularity", Proc. Of the Royal Society B, 280: 20122863.

(5) S. A. Ehmann and M. C Lin, "Accurate and Fast Proximity Queries between Polyhedra Using Surface Decomposition", Computer Graphics Forum, 2001

(6) www.blendswap.com/

(7) V. Parque, M. Kobayashi and M. Higashi, "Explorit for Global Optimization", NIPS Workshop on Optimization for Machine Learning, Lake Tahoe, Nevada, USA, 2012. 